\title{
A New Method with a Different Auxiliary Equation to Obtain Solitary Wave Solutions for Nonlinear Partial Differential Equations
}

\author{
Bülent Kiliç and Hasan Bulut \\ Department of Mathematics, Firat University, 23119 Elazig, Turkey \\ Correspondence should be addressed to Hasan Bulut; hbulut@firat.edu.tr
}

Received 3 February 2013; Revised 22 April 2013; Accepted 23 April 2013

Academic Editor: Dumitru Baleanu

Copyright @ 2013 B. Kiliç and H. Bulut. This is an open access article distributed under the Creative Commons Attribution License, which permits unrestricted use, distribution, and reproduction in any medium, provided the original work is properly cited.

A new method with a different auxiliary equation from the Riccati equation is used for constructing exact travelling wave solutions of nonlinear partial differential equations. The main idea of this method is to take full advantage of a different auxilliary equation from the Riccati equation which has more new solutions. More new solitary solutions are obtained for the RLW Burgers and Hirota Satsuma coupled equations.

\section{Introduction}

In the recent years, remarkable progress has been made in the construction of the exact solutions for nonlinear partial differential equations, which have been a basic concern for both mathematicians and physicists [1-3].We do not attempt to characterize the general form of nonlinear dispersive wave equations $[4,5]$. When an original nonlinear equation is directly calculated, the solution will preserve the actual physical characters of solutions [6]. The studies in finding exact solutions to nonlinear differential equation (NPDE), when they exist, are very important for the understanding of most nonlinear physical phenomena. There are many studies which obtain explicit solutions for nonlinear differential equations. Many explicit exact methods have been introduced in literature [7-21]. Some of them are generalized Miura transformation, Darboux transformation, Cole-Hopf transformation, Hirota's dependent variable transformation, the inverse scattering transform and the Bäcklund transformation, tanh method, sine-cosine method, Painleve method, homogeneous balance method (HB), similarity reduction method, improved tanh method and so on.

In this article, the first section presents the scope of the study as an introduction. In the second section contains analyze of a new method and balance term definition. In the third section, we will obtain wave solutions of RLW Burgers and Hirota Satsuma coupled equations by using a new method. In the last section, we implement the conclusion.

\section{Method and Its Applications}

Let us simply describe the method [22]. Consider a given partial differential equation in two variables

$$
H\left(u, u_{t}, u_{x}, u_{x x}, \ldots\right)=0 .
$$

The fact that the solutions of many nonlinear equations can be expressed as a finite series of solutions of the auxiliary equation motivates us to seek for the solutions of (1) in the form

$$
u(x, t)=\lambda \sum_{i=0}^{m}\left[a_{i} F(\xi)^{i}+a_{-i} F(\xi)^{-i}\right]
$$

where, $\xi=k(x-c t), k$ and $c$ are the wave number and the wave speed respectively, $m$ is a positive integer that can be determined by balancing the linear term of highest order with the nonlinear term in (1), $\lambda$ is balancing coefficient that will be defined in a new "Balance term" definition and $a_{0}, a_{1}, a_{2}, \ldots$ are parameters to be determined. Substituting (2) into (1) yields a set of algebraic equations for $a_{0}, a_{1}, a_{2}, \ldots$ because all coefficients of $F$ have to vanish. From these 


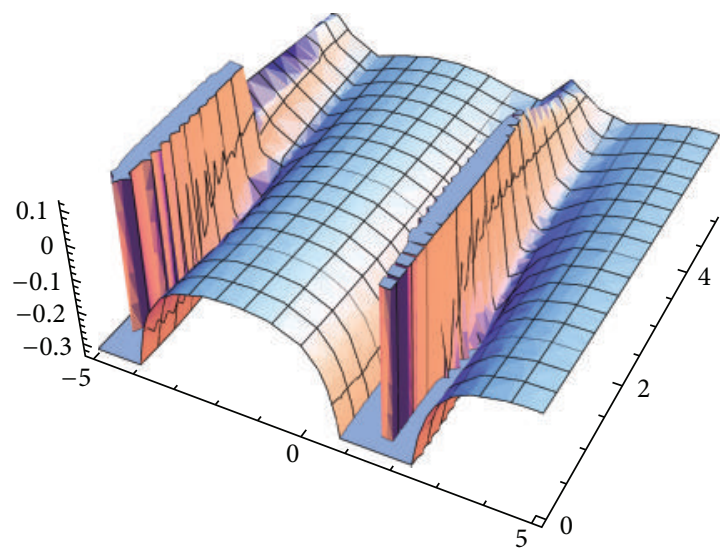

(a)

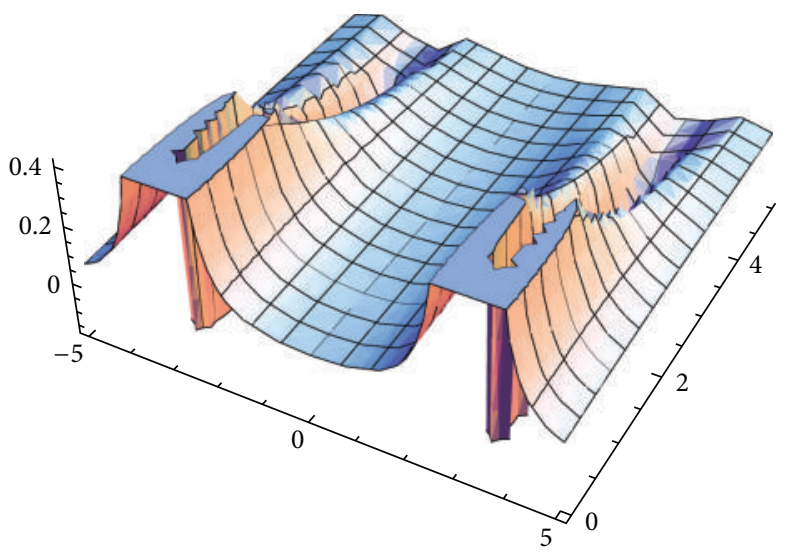

(b)

FIGURE 1: Graph of the solution $u(x, t)$ from left to the right for (13) and (14).

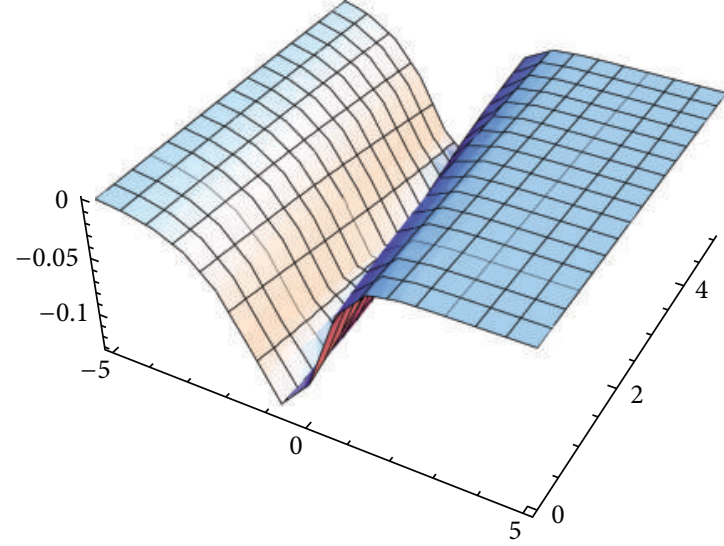

(a)

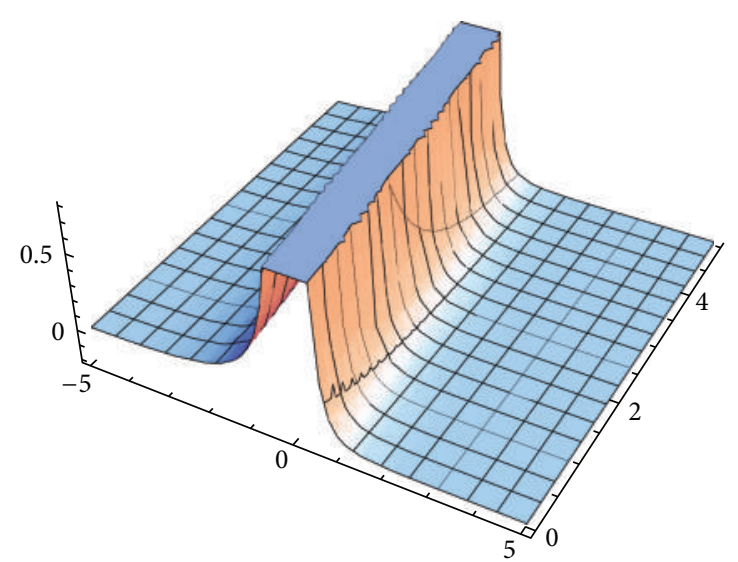

(b)

FIGURE 2: Graph of the solution $u(x, t)$ from left to the right for (15) and (16).

relations $a_{0}, a_{1}, a_{2}, \ldots$ can be determined. The main idea of our method is to take full advantage of the new auxiliary equation. The desired auxiliary equation presents as following

$$
F^{\prime}=\frac{A}{F}+B F+C F^{3}
$$

where $d F / d \xi=F^{\prime}$ and $A, B, C$ are constants.

Case 1. If $A=-1 / 4, B=1 / 2, C=-1 / 2$ then (3) has the solution $F=1 / \sqrt{1+\tan (\xi)+\sec (\xi)}$.

Case 2. If $A=1 / 4, B=-1 / 2, C=0$ then (3) has the solutions $F=1 / \sqrt{1+\operatorname{csch}(\xi)+\operatorname{coth}(\xi)}$ or $F=$ $1 / \sqrt{1+i \operatorname{sech}(\xi)+\tanh (\xi)}$.

Case 3. If $A=1 / 2, B=-1, C=0$ then (3) has the solutions $F=1 / \sqrt{1+\cot h(\xi)}$ or

$$
F=\frac{1}{\sqrt{1+\tanh (\xi)}}
$$

Remark 1. Depending on the $A, B$ and $C$ coefficients in the (3), it could be reached only three cases.

In the following we present a new approach to the "Balance term" definition.

Definition 2. When (1) is transformed with $u(x, t)=u(\xi)$, $\xi=k(x-c t)$, where $k$ and $c$ are real constants, we get a nonlinear ordinary differential equation for $u(\xi)$ as following

$$
Q^{\prime}\left(u, k c u^{\prime}, k u^{\prime}, k^{2} u^{\prime \prime}, \ldots\right)=0
$$

Let $u^{(p)}$ is the highest order derivative linear term and $u^{q} u^{(r)}$ is the highest nonlinear term in (5) and $F^{\prime}=k_{0}+k_{1} F+$ $k_{2} F^{2}+\cdots+k_{n} F^{n}$ is the auxiliary equation that is used to solve the nonlinear partial differential equation then the "Balance term" $m$ can be decided by the balancing the nonlinear term $u^{q} u^{(r)}$ and the linear term $u^{(p)}$ with acceptances of $u \cong \lambda F^{i}$ and $F^{\prime} \cong F^{n}$ where $n$ is integer $(n \neq 1)$ and $\lambda$ is the balance coefficient that can be determined later. 


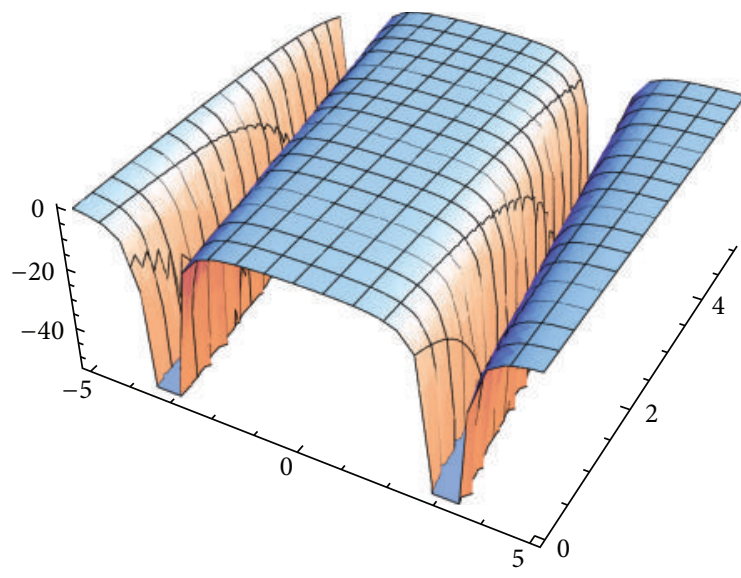

(a)

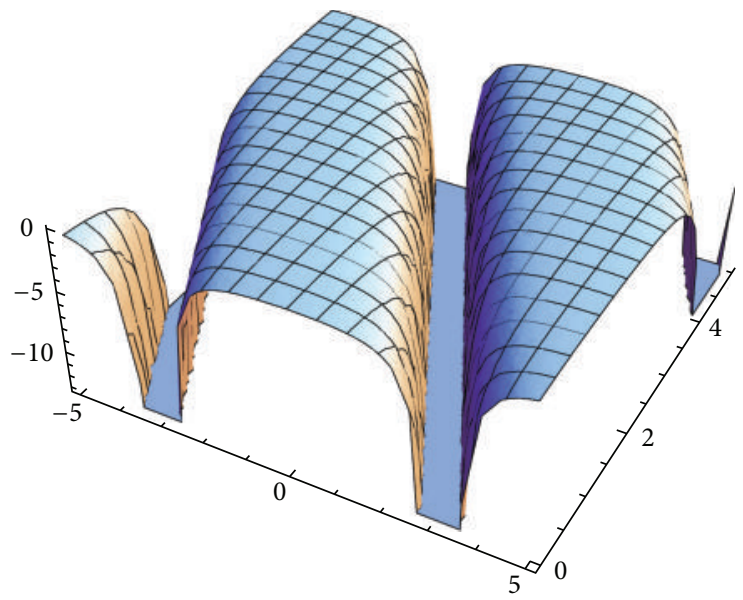

(c)

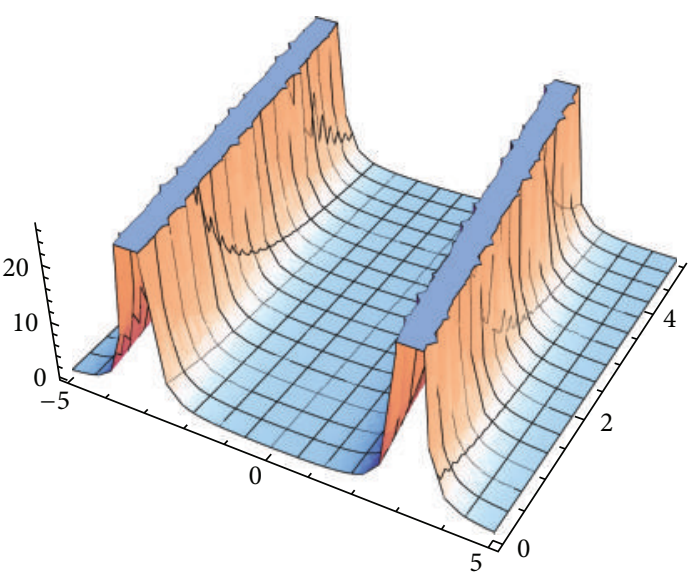

(b)

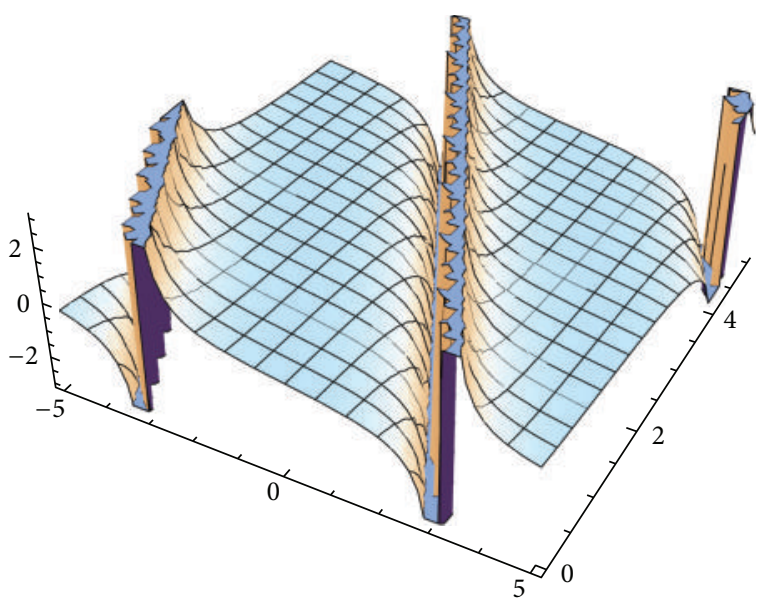

(d)

FIGURE 3: Graph of the solutions $u(x, t)$ and $v(x, t)$ corresponding to the value $b_{0}=1$ from left to the right for (22), (3), and (23).

Example 1. For the $\mathrm{KdV}$ equation with the transform $u(x, t)=u(\xi), \xi=x-c t$ we have the ordinary differential equation as following

$$
-c u^{\prime}+6 u u^{\prime}+u^{\prime \prime \prime}=0 \text {. }
$$

By the balancing linear term $u^{\prime \prime \prime}$ with nonlinear term $u u^{\prime}$

$$
\begin{gathered}
u^{\prime}=\left(\lambda F^{m}\right)^{\prime}=\lambda m F^{m-1} F^{\prime}=\lambda m F^{m-1} F^{n}=\lambda m F^{m+n-1}, \\
u^{\prime \prime}=\left(\lambda m F^{m+n-1}\right)^{\prime}=\lambda m(m+n-1) F^{m+n-2} F^{\prime} \\
=\lambda m(m+n-1) F^{m+n-2} F^{n}=\lambda m(m+n-1) F^{m+2 n-2}, \\
u^{\prime \prime \prime}=\left(\lambda m(m+n-1) F^{m+2 n-2}\right)^{\prime} \\
=\lambda m(m+n-1)(m+2 n-2) F^{m+2 n-3} F^{\prime} \\
=\lambda m(m+n-1)(m+2 n-2) F^{m+2 n-3} F^{n} \\
=\lambda m(m+n-1)(m+2 n-2) F^{m+3 n-3}, \\
u u^{\prime}=\lambda F^{m} \lambda m F^{m+n-1}=\lambda^{2} m F^{2 m+n-1}
\end{gathered}
$$

we have the equations above and the equating $u u^{\prime}$ to $u^{\prime \prime \prime}$

$$
\begin{gathered}
\lambda^{2} m F^{2 m+n-1}=\lambda m(m+n-1)(m+2 n-2) F^{m+3 n-3}, \\
\lambda=(m+n-1)(m+2 n-2), \quad m=2(n-1) .
\end{gathered}
$$

If it is noticed that our new balance term $m(m=2(n-1))$ is connected to $n$. Namely our new balance term definition is connected to chosen auxiliary equation.

\section{Application of the Method}

Example 2. Let's consider RLW Burgers equation

$$
u_{t}+u_{x}+12 u u_{x}-u_{x x}-u_{x x t}=0 \text {, }
$$

with the transform $u(x, t)=u(\xi), \xi=x-c t$ we have the following equation

$$
-c u^{\prime}+u^{\prime}+12 u u^{\prime}-u^{\prime \prime}+c u^{\prime \prime \prime}=0 .
$$




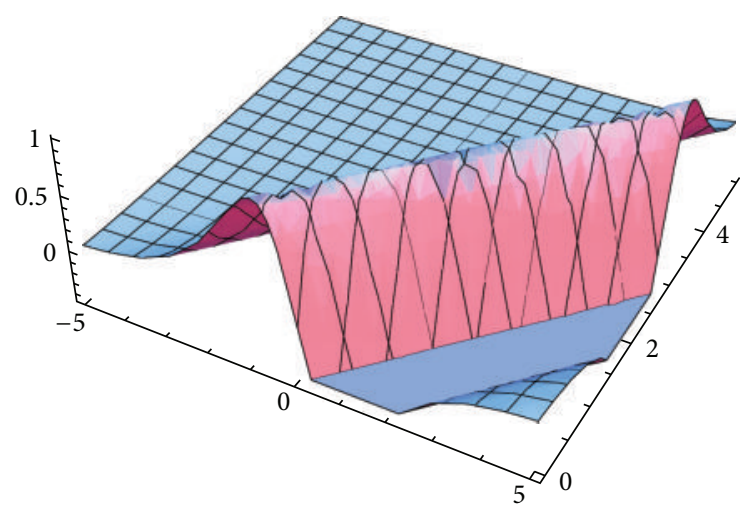

(a)

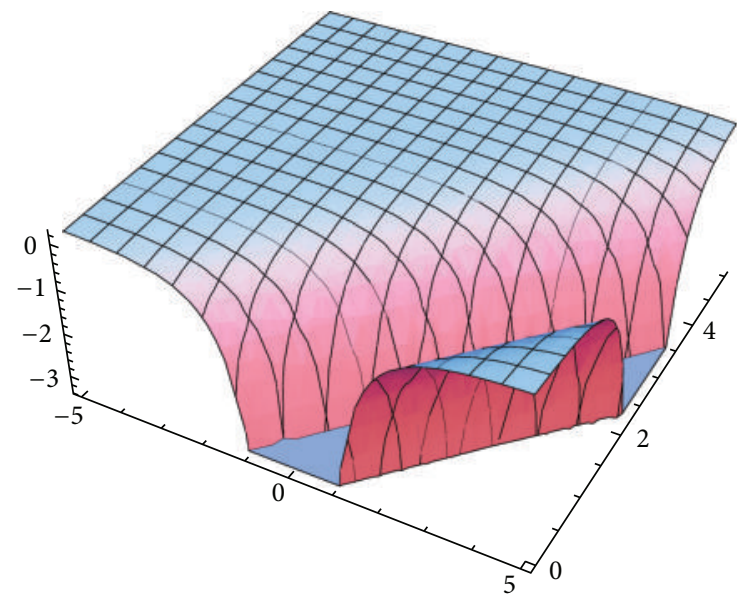

(c)

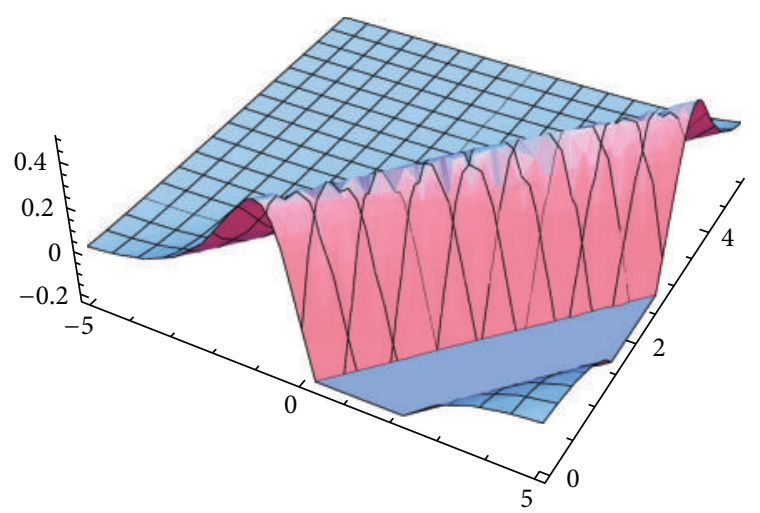

(b)

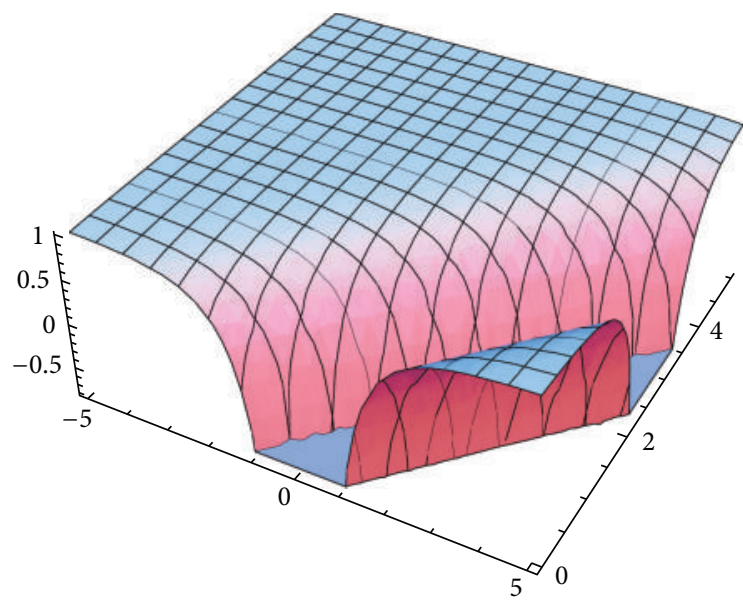

(d)

FIGURE 4: Graph of the solutions $u(x, t)$ and $v(x, t)$ corresponding to the value $b_{0}=1$ from left to the right for (25) and (26).

From the Definition 2 we have the balance term of RLW Burgers equation by using the auxiliary equation (3) for "Case 1", is equal to 4 . Therefore, we may choose the following ansatz:

$$
\begin{array}{r}
u(x, t)=48\left(a_{-4} F^{-4}+a_{-3} F^{-3}+a_{-2} F^{-2}+a_{-1} F^{-1}\right. \\
\left.+a_{0}+a_{1} F+a_{2} F^{2}+a_{3} F^{3}+a_{4} F^{4}\right) .
\end{array}
$$

Substituting (11) into (10) along with (3) and using Mathematica yields a system of equations w.r.t. $F^{i}$. Setting the coefficients of $F^{i}$ in the obtained system of equations to zero, we can deduce the following set of algebraic polynomials with the respect unknowns $a_{0}, a_{1}, a_{2}, \ldots$ for the Case 1 :

$$
\begin{gathered}
3 c a_{-4}+12 a_{-4}^{2}=0, \\
\left(\frac{105 c a_{-3}}{64}+21 a_{-4} a_{-3}\right)=0,
\end{gathered}
$$

$$
\begin{gathered}
\left(-\frac{3 a_{-4}}{2}-\frac{27 c a_{-4}}{2}-24 a_{-4}^{2}+9 a_{-3}^{2}\right. \\
\left.+\frac{3 c a_{-2}}{4}+18 a_{-4} a_{-2}\right)=0 \\
\left(-\frac{15 a_{-3}}{16}-\frac{225 c a_{-3}}{32}-42 a_{-4} a_{-3}+15 a_{-3} a_{-2}\right. \\
\left.+\frac{15 c a_{-1}}{64}+15 a_{-4} a_{-1}\right)=0 \\
\left(6 a_{-4}+28 c a_{-4}+24 a_{-4}^{2}-18 a_{-3}^{2}-\frac{a_{-2}}{2}-3 c a_{-2}\right. \\
\left.-36 a_{-4} a_{-2}+6 a_{-2}^{2}+12 a_{-3} a_{-1}+12 a_{-4} a_{0}\right)=0 \\
\left(\frac{15 a_{-3}}{4}+\frac{429 c a_{-3}}{32}+42 a_{-4} a_{-3}-30 a_{-3} a_{-2}\right. \\
-\frac{3 a_{-1}}{16}-\frac{27 c a_{-1}}{32}-30 a_{-4} a_{-1}+9 a_{-2} a_{-1} \\
\left.+9 a_{-3} a_{0}-\frac{3 c a_{1}}{64}+9 a_{-4} a_{1}\right)=0
\end{gathered}
$$




$$
\begin{aligned}
& \left(8 a_{-4}+20 c a_{-4}-3 a_{-2}-4 c a_{-2}+12 a_{-2}^{2}\right. \\
& +24 a_{-3} a_{-1}-6 a_{-1}^{2}+24 a_{-4} a_{0} \\
& \left.-12 a_{-2} a_{0}-12 a_{-3} a_{1}-12 a_{-4} a_{2}\right)=0, \\
& \left(-10 a_{-4}-32 c a_{-4}+18 a_{-3}^{2}+2 a_{-2}\right. \\
& +5 c a_{-2}+36 a_{-4} a_{-2}-12 a_{-2}^{2} \\
& -24 a_{-3} a_{-1}+3 a_{-1}^{2}-24 a_{-4} a_{0} \\
& \left.+6 a_{-2} a_{0}+6 a_{-3} a_{1}+6 a_{-4} a_{2}\right)=0, \\
& \left(-6 a_{-3}-\frac{27 c a_{-3}}{2}+30 a_{-3} a_{-2}+\frac{3 a_{-1}}{4}\right. \\
& +\frac{35 c a_{-1}}{32}+30 a_{-4} a_{-1}-18 a_{-2} a_{-1} \\
& -18 a_{-3} a_{0}+3 a_{-1} a_{0}+\frac{a_{1}}{16}+\frac{3 c a_{1}}{32} \\
& -18 a_{-4} a_{1}+3 a_{-2} a_{1}+3 a_{-3} a_{2} \\
& \left.+\frac{3 c a_{3}}{64}+3 a_{-4} a_{3}\right)=0 \text {, } \\
& \left(\frac{9 a_{-3}}{2}+\frac{105 c a_{-3}}{16}-a_{-1}-\frac{c a_{-1}}{2}+18 a_{-2} a_{-1}\right. \\
& +18 a_{-3} a_{0}-6 a_{-1} a_{0}-\frac{a_{1}}{4}+\frac{c a_{1}}{32} \\
& +18 a_{-4} a_{1}-6 a_{-2} a_{1}-3 a_{0} a_{1} \\
& -6 a_{-3} a_{2}-3 a_{-1} a_{2}-\frac{3 a_{3}}{16}+\frac{9 c a_{3}}{32} \\
& \left.-6 a_{-4} a_{3}-3 a_{-2} a_{3}-3 a_{-3} a_{4}\right)=0, \\
& \left(-2 a_{-4}-6 c a_{-4}+2 a_{-2}+c a_{-2}+6 a_{-1}^{2}\right. \\
& +12 a_{-2} a_{0}+12 a_{-3} a_{1}-3 a_{1}^{2}-\frac{c a_{2}}{2} \\
& +12 a_{-4} a_{2}-6 a_{0} a_{2}-6 a_{-1} a_{3}-\frac{a_{4}}{2} \\
& \left.+\frac{3 c a_{4}}{2}-6 a_{-2} a_{4}\right)=0 \\
& \left(-\frac{3 a_{-3}}{4}-\frac{9 c a_{-3}}{8}+\frac{a_{-1}}{2}-\frac{c a_{-1}}{16}+6 a_{-1} a_{0}\right. \\
& +\frac{c a_{1}}{2}+6 a_{-2} a_{1}+6 a_{0} a_{1}+6 a_{-3} a_{2} \\
& +6 a_{-1} a_{2}-9 a_{1} a_{2}+\frac{3 a_{3}}{4}-\frac{105 c a_{3}}{32} \\
& +6 a_{-4} a_{3}+6 a_{-2} a_{3}-9 a_{0} a_{3} \\
& \left.+6 a_{-3} a_{4}-9 a_{-1} a_{4}\right)=0,
\end{aligned}
$$$$
\left(6 a_{1}^{2}-a_{2}+4 c a_{2}+12 a_{0} a_{2}-6 a_{2}^{2}\right.
$$$$
+12 a_{-1} a_{3}-12 a_{1} a_{3}+2 a_{4}-10 c a_{4}
$$$$
\left.+12 a_{-2} a_{4}-12 a_{0} a_{4}\right)=0,
$$$$
\left(-\frac{3}{8} c a_{-3}+\frac{a_{-1}}{4}-\frac{3 c a_{-1}}{8}+\frac{a_{1}}{2}-\frac{35 c a_{1}}{16}\right.
$$$$
-6 a_{0} a_{1}-6 a_{-1} a_{2}+18 a_{1} a_{2}-3 a_{3}
$$$$
+\frac{27 c a_{3}}{2}-6 a_{-2} a_{3}+18 a_{0} a_{3}-15 a_{2} a_{3}
$$$$
\left.-6 a_{-3} a_{4}+18 a_{-1} a_{4}-15 a_{1} a_{4}\right)=0,
$$$$
\left(-6 a_{1}^{2}+2 a_{2}-10 c a_{2}-12 a_{0} a_{2}+12 a_{2}^{2}\right.
$$$$
-12 a_{-1} a_{3}+24 a_{1} a_{3}-9 a_{3}^{2}-6 a_{4}+32 c a_{4}
$$$$
\left.-12 a_{-2} a_{4}+24 a_{0} a_{4}-18 a_{2} a_{4}\right)=0,
$$$$
\left(-\frac{105 c a_{3}}{8}-42 a_{3} a_{4}\right)=0,
$$$$
\left(\frac{3 c a_{-1}}{8}-\frac{3 a_{1}}{4}+\frac{27 c a_{1}}{8}-18 a_{1} a_{2}+\frac{9 a_{3}}{2}\right.
$$$$
-\frac{429 c a_{3}}{16}-18 a_{0} a_{3}+30 a_{2} a_{3}
$$$$
\left.-18 a_{-1} a_{4}+30 a_{1} a_{4}-21 a_{3} a_{4}\right)=0,
$$$$
\left(-\frac{15 c a_{1}}{8}-\frac{15 a_{3}}{4}+\frac{225 c a_{3}}{8}\right.
$$$$
\left.-30 a_{2} a_{3}-30 a_{1} a_{4}+42 a_{3} a_{4}\right)=0,
$$$$
\left(-24 c a_{4}-24 a_{4}^{2}\right)=0,
$$$$
\left(-2 a_{2}+12 c a_{2}-12 a_{2}^{2}-24 a_{1} a_{3}\right.
$$$$
+18 a_{3}^{2}+8 a_{4}-56 c a_{4}-24 a_{0} a_{4}
$$$$
\left.+36 a_{2} a_{4}-12 a_{4}^{2}\right)=0,
$$$$
\left(-6 c a_{2}-18 a_{3}^{2}-6 a_{4}\right.
$$$$
\left.+54 c a_{4}-36 a_{2} a_{4}+24 a_{4}^{2}\right)=0 .
$$

From the system of (12) we have

$$
\begin{gathered}
a_{0}=-\frac{11}{60}-\frac{i}{12}, \\
a_{1}=a_{2}=a_{3}=a_{4}=a_{-1}=a_{-3}=0,
\end{gathered}
$$




$$
\begin{gathered}
a_{-2}=\frac{1}{10}+\frac{i}{10}, \\
a_{-4}=-\frac{i}{20}, \quad c=\frac{i}{5}, \\
u(x, t) \\
=-\left(\frac{11+5 i}{60}\right)+\left(\frac{1+i}{10}\right) \\
\times\left(\frac{i}{\sqrt{1+\sec [x-(i / 5) t]+\tan [x-(i / 5) t]}}\right)^{-2} \\
-\frac{1}{20}\left(\frac{1}{\sqrt{1+\sec [x-(i / 5) t]+\tan [x-(i / 5) t]}}\right)^{-4} .
\end{gathered}
$$

(ii)

$$
\begin{gathered}
a_{0}=\frac{1+5 i}{60}, \quad c=-a_{4}, \\
a_{1}=a_{-2}=a_{3}=a_{-4}=a_{-1}=a_{-3}=0 \\
a_{2}=-\frac{1+i}{5}, \quad a_{4}= \pm \frac{i}{5} \\
u(x, t)=\frac{1+5 i}{60}-\frac{1+i}{5} \\
\times\left(\sqrt{1+\sec \left[x+\frac{i}{5} t\right]+\tan \left[x+\frac{i}{5} t\right]}\right)^{2} \\
+\frac{i}{5}\left(\sqrt{1+\sec \left[x+\frac{i}{5} t\right]+\tan \left[x+\frac{i}{5} t\right]}\right)^{4} .
\end{gathered}
$$

From the Definition 2 we have the balance term of RLW Burgers equation by using the auxiliary equation (3) for "Case 2 ", is equal to -4 then we have the following system of equations

$$
\begin{gathered}
-3 c a_{-4}-12 a_{-4}^{2}=0 \\
\left(-\frac{3 a_{-4}}{2}+\frac{27 c a_{-4}}{2}+24 a_{-4}^{2}-9 a_{-3}^{2}\right. \\
\left.-\frac{3 c a_{-2}}{4}-18 a_{-4} a_{-2}\right)=0 \\
\left(-\frac{15 a_{-3}}{16}+\frac{225 c a_{-3}}{32}+42 a_{-4} a_{-3}-15 a_{-3} a_{-2}\right. \\
\left.-\frac{15 c a_{-1}}{64}-15 a_{-4} a_{-1}\right)=0, \\
\left(4 a_{-4}-18 c a_{-4}+18 a_{-3}^{2}-\frac{a_{-2}}{2}\right. \\
+3 c a_{-2}+36 a_{-4} a_{-2}-6 a_{-2}^{2} \\
\left.-12 a_{-3} a_{-1}-12 a_{-4} a_{0}\right)=0
\end{gathered}
$$

$$
\begin{gathered}
\left(\frac{9 a_{-3}}{4}-\frac{135 c a_{-3}}{16}+30 a_{-3} a_{-2}\right. \\
-\frac{3 a_{-1}}{16}+\frac{27 c a_{-1}}{32}+30 a_{-4} a_{-1} \\
\left.-9 a_{-2} a_{-1}-9 a_{-3} a_{0}\right)=0 \\
\left(-2 a_{-4}+6 c a_{-4}+a_{-2}-3 c a_{-2}\right. \\
+12 a_{-2}^{2}+24 a_{-3} a_{-1}-3 a_{-1}^{2} \\
\left.+24 a_{-4} a_{0}-6 a_{-2} a_{0}\right)=0 \\
\left(-\frac{3 a_{-3}}{4}+\frac{15 c a_{-3}}{8}+\frac{a_{-1}}{4}-\frac{9 c a_{-1}}{16}\right. \\
\left.+18 a_{-2} a_{-1}+18 a_{-3} a_{0}-3 a_{-1} a_{0}\right)=0 \\
\left(-\frac{105}{64} c a_{-3}-21 a_{-4} a_{-3}\right)=0 \\
\left(6 a_{-1}^{2}+12 a_{-2} a_{0}\right)=0 \\
\left(\frac{a_{-1}}{4}-\frac{3 c a_{-1}}{8}+6 a_{-1} a_{0}\right)=0 .
\end{gathered}
$$

From the system of (15) we have

(i)

$$
\begin{gathered}
a_{0}=a_{-1}=0, \quad a_{-2}=-\frac{1}{5}, \\
a_{-3}=0, \quad a_{-4}=\frac{1}{20}, \quad c=-\frac{1}{5},
\end{gathered}
$$

$u(x, t)$

$$
\begin{aligned}
= & -\frac{1}{5}\left(\frac{1}{\sqrt{1+i \operatorname{sech}[x+(1 / 5) t]+\tanh [x+(1 / 5) t]}}\right)^{-2} \\
& +\frac{1}{20}\left(\frac{1}{\sqrt{1+i \operatorname{sech}[x+(1 / 5) t]+\tanh [x+(1 / 5) t]}}\right)^{-4},
\end{aligned}
$$

or

$$
\begin{aligned}
& u(x, t) \\
& =-\frac{1}{5}\left(\frac{1}{\sqrt{1+\operatorname{csch}[x+(1 / 5) t]+\operatorname{coth}[x+(1 / 5) t]}}\right)^{-2} \\
& \quad+\frac{1}{20}\left(\frac{1}{\sqrt{1+\operatorname{csch}[x+(1 / 5) t]+\operatorname{coth}[x+(1 / 5) t]}}\right)^{-4} .
\end{aligned}
$$


Example 3. Let's consider Hirota Satsuma coupled equation

$$
\begin{gathered}
u_{t}-3 u u_{x}+6 v v_{x}-\frac{1}{2} u_{x x x}=0, \\
v_{t}+3 u v_{x}+v_{x x x}=0
\end{gathered}
$$

with the transform $u(x, t)=u(\xi), v(x, t)=v(\xi), \xi=x-c t$ we have

$$
\begin{gathered}
-c u^{\prime}-3 u u^{\prime}+6 v v^{\prime}-\frac{1}{2} u^{\prime \prime \prime}=0, \\
-c v^{\prime}+3 u v^{\prime}+v^{\prime \prime \prime}=0 .
\end{gathered}
$$

From the Definition 2 we have the balance term of Hirota Satsuma coupled equation by using the auxiliary equation (3) for "Case 1 ", is equal to 4 for $u$ and $v$. Therefore, we may choose the following ansatz:

$$
\begin{aligned}
& u(x, t)=48\left(a_{0}+a_{1} F+a_{2} F^{2}+a_{3} F^{3}+a_{4} F^{4}\right), \\
& v(x, t)=48\left(b_{0}+b_{1} F+b_{2} F^{2}+b_{3} F^{3}+b_{4} F^{4}\right) .
\end{aligned}
$$

Substituting (20) into (19) along with (3) and using Mathematica yields a system of equations w.r.t. $F^{i}$. Setting the coefficients of $F^{i}$ in the obtained system of equations to zero, we can deduce the following set of algebraic polynomials with the respect unknowns $a_{0}, a_{1}, a_{2}, \ldots$ for the Case 1 :

$$
\begin{gathered}
\frac{3 a_{1}}{128}=0 \\
\left(-\frac{3 a_{1}}{64}-\frac{3 a_{3}}{128}\right)=0 \\
\left(\frac{7 a_{1}}{64}+\frac{c a_{1}}{4}+\frac{3 a_{0} a_{1}}{4}-\frac{9 a_{3}}{64}-\frac{3 b_{0} b_{1}}{2}\right)=0 \\
\left(\frac{3 a_{1}^{2}}{4}+\frac{a_{2}}{2}+\frac{c a_{2}}{2}+\frac{3 a_{0} a_{2}}{2}\right. \\
\left.-\frac{3 a_{4}}{4}-\frac{3 b_{1}^{2}}{2}-3 b_{0} b_{2}\right)=0
\end{gathered}
$$

$$
\begin{aligned}
& \left(-\frac{a_{1}}{2}-\frac{c a_{1}}{2}-\frac{3 a_{0} a_{1}}{2}+\frac{9 a_{1} a_{2}}{4}\right. \\
& +\frac{129 a_{3}}{64}+\frac{3 c a_{3}}{4}+\frac{9 a_{0} a_{3}}{4} \\
& \left.+3 b_{0} b_{1}-\frac{9 b_{1} b_{2}}{2}-\frac{9 b_{0} b_{3}}{2}\right)=0, \\
& \left(-\frac{3 a_{1}^{2}}{2}-\frac{5 a_{2}}{2}-c a_{2}-3 a_{0} a_{2}\right. \\
& +\frac{3 a_{2}^{2}}{2}+3 a_{1} a_{3}+\frac{11 a_{4}}{2}+c a_{4} \\
& +3 a_{0} a_{4}+3 b_{1}^{2}+6 b_{0} b_{2}-3 b_{2}^{2} \\
& \left.-6 b_{1} b_{3}-6 b_{0} b_{4}\right)=0, \\
& \left(\frac{43 a_{1}}{32}+\frac{c a_{1}}{2}+\frac{3 a_{0} a_{1}}{2}-\frac{9 a_{1} a_{2}}{2}\right. \\
& -\frac{15 a_{3}}{2}-\frac{3 c a_{3}}{2}-\frac{9 a_{0} a_{3}}{2}+\frac{15 a_{2} a_{3}}{4} \\
& +\frac{15 a_{1} a_{4}}{4}-3 b_{0} b_{1}+9 b_{1} b_{2} \\
& \left.+9 b_{0} b_{3}-\frac{15 b_{2} b_{3}}{2}-\frac{15 b_{1} b_{4}}{2}\right)=0, \\
& \left(\frac{3 a_{1}^{2}}{2}+\frac{11 a_{2}}{2}+c a_{2}+3 a_{0} a_{2}-3 a_{2}^{2}\right. \\
& -6 a_{1} a_{3}+\frac{9 a_{3}^{2}}{4}-17 a_{4}-2 c a_{4} \\
& -6 a_{0} a_{4}+\frac{9 a_{2} a_{4}}{2}-3 b_{1}^{2}-6 b_{0} b_{2} \\
& +6 b_{2}^{2}+12 b_{1} b_{3}-\frac{9 b_{3}^{2}}{2} \\
& \left.+12 b_{0} b_{4}-9 b_{2} b_{4}\right)=0 \text {, } \\
& \left(\frac{105 a_{3}}{16}+\frac{21 a_{3} a_{4}}{2}-21 b_{3} b_{4}\right)=0, \\
& \left(-\frac{27 a_{1}}{16}+\frac{9 a_{1} a_{2}}{2}+\frac{453 a_{3}}{32}+\frac{3 c a_{3}}{2}\right. \\
& +\frac{9 a_{0} a_{3}}{2}-\frac{15 a_{2} a_{3}}{2}-\frac{15 a_{1} a_{4}}{2} \\
& +\frac{21 a_{3} a_{4}}{4}-9 b_{1} b_{2}-9 b_{0} b_{3} \\
& \left.+15 b_{2} b_{3}+15 b_{1} b_{4}-\frac{21 b_{3} b_{4}}{2}\right)=0,
\end{aligned}
$$




$$
\begin{aligned}
& \left(\frac{15 a_{1}}{16}-\frac{225 a_{3}}{16}+\frac{15 a_{2} a_{3}}{2}\right. \\
& +\frac{15 a_{1} a_{4}}{2}-\frac{21 a_{3} a_{4}}{2}-15 b_{2} b_{3} \\
& \left.-15 b_{1} b_{4}+21 b_{3} b_{4}\right)=0, \\
& \left(12 a_{4}+6 a_{4}^{2}-12 b_{4}^{2}\right)=0, \\
& \left(-6 a_{2}+3 a_{2}^{2}+6 a_{1} a_{3}-\frac{9 a_{3}^{2}}{2}\right. \\
& +29 a_{4}+2 c a_{4}+6 a_{0} a_{4} \\
& -9 a_{2} a_{4}+3 a_{4}^{2}-6 b_{2}^{2} \\
& -12 b_{1} b_{3}+9 b_{3}^{2}-12 b_{0} b_{4} \\
& \left.+18 b_{2} b_{4}-6 b_{4}^{2}\right)=0-\frac{3 b_{1}}{64}=0, \\
& \left(\frac{3 b_{1}}{32}+\frac{3 b_{3}}{64}\right)=0, \\
& \left(-\frac{7 b_{1}}{32}+\frac{c b_{1}}{4}-\frac{3 a_{0} b_{1}}{4}+\frac{9 b_{3}}{32}\right)=0, \\
& \left(-\frac{3}{4} a_{1} b_{1}-b_{2}+\frac{c b_{2}}{2}-\frac{3 a_{0} b_{2}}{2}+\frac{3 b_{4}}{2}\right)=0, \\
& \left(b_{1}-\frac{c b_{1}}{2}+\frac{3 a_{0} b_{1}}{2}-\frac{3 a_{2} b_{1}}{4}-\frac{3 a_{1} b_{2}}{2}\right. \\
& \left.-\frac{129 b_{3}}{32}+\frac{3 c b_{3}}{4}-\frac{9 a_{0} b_{3}}{4}\right)=0, \\
& \left(\frac{3 a_{1} b_{1}}{2}-\frac{3 a_{3} b_{1}}{4}+5 b_{2}-c b_{2}\right. \\
& +3 a_{0} b_{2}-\frac{3 a_{2} b_{2}}{2}-\frac{9 a_{1} b_{3}}{4} \\
& \left.-11 b_{4}+c b_{4}-3 a_{0} b_{4}\right)=0, \\
& \left(-\frac{43 b_{1}}{16}+\frac{c b_{1}}{2}-\frac{3 a_{0} b_{1}}{2}+\frac{3 a_{2} b_{1}}{2}\right. \\
& -\frac{3 a_{4} b_{1}}{4}+3 a_{1} b_{2}-\frac{3 a_{3} b_{2}}{2}+15 b_{3} \\
& \left.-\frac{3 c b_{3}}{2}+\frac{9 a_{0} b_{3}}{2}-\frac{9 a_{2} b_{3}}{4}-3 a_{1} b_{4}\right)=0, \\
& \left(-\frac{3}{2} a_{1} b_{1}+\frac{3 a_{3} b_{1}}{2}-11 b_{2}+c b_{2}\right. \\
& -3 a_{0} b_{2}+3 a_{2} b_{2}-\frac{3 a_{4} b_{2}}{2} \\
& +\frac{9 a_{1} b_{3}}{2}-\frac{9 a_{3} b_{3}}{4}+34 b_{4}
\end{aligned}
$$

$$
\begin{aligned}
& \left.-2 c b_{4}+6 a_{0} b_{4}-3 a_{2} b_{4}\right)=0, \\
& \left(-\frac{105 b_{3}}{8}-\frac{9 a_{4} b_{3}}{2}-6 a_{3} b_{4}\right)=0, \\
& \left(\frac{27 b_{1}}{8}-\frac{3 a_{2} b_{1}}{2}+\frac{3 a_{4} b_{1}}{2}-3 a_{1} b_{2}\right. \\
& +3 a_{3} b_{2}-\frac{453 b_{3}}{16}+\frac{3 c b_{3}}{2}-\frac{9 a_{0} b_{3}}{2} \\
& \left.+\frac{9 a_{2} b_{3}}{2}-\frac{9 a_{4} b_{3}}{4}+6 a_{1} b_{4}-3 a_{3} b_{4}\right)=0, \\
& \left(-\frac{15 b_{1}}{8}-\frac{3 a_{4} b_{1}}{2}-3 a_{3} b_{2}+\frac{225 b_{3}}{8}\right. \\
& \left.-\frac{9 a_{2} b_{3}}{2}+\frac{9 a_{4} b_{3}}{2}-6 a_{1} b_{4}+6 a_{3} b_{4}\right)=0 \\
& \left(-24 b_{4}-6 a_{4} b_{4}\right)=0, \\
& \left(-\frac{3}{2} a_{3} b_{1}+12 b_{2}-3 a_{2} b_{2}+3 a_{4} b_{2}\right. \\
& -\frac{9 a_{1} b_{3}}{2}+\frac{9 a_{3} b_{3}}{2}-58 b_{4}+2 c b_{4} \\
& \left.-6 a_{0} b_{4}+6 a_{2} b_{4}-3 a_{4} b_{4}\right)=0, \\
& \left(-6 b_{2}-3 a_{4} b_{2}-\frac{9 a_{3} b_{3}}{2}+54 b_{4}\right. \\
& \left.-6 a_{2} b_{4}+6 a_{4} b_{4}\right)=0 \\
& \left(3 a_{2}+\frac{9 a_{3}^{2}}{2}-27 a_{4}+9 a_{2} a_{4}-6 a_{4}^{2}\right. \\
& \left.-9 b_{3}^{2}-18 b_{2} b_{4}+12 b_{4}^{2}\right)=0 \text {. }
\end{aligned}
$$

From the system of (21) we have

$$
\begin{aligned}
c=\frac{1}{4}\left(5-6 b_{0}\right), & a_{0}=\frac{1}{4}\left(-5-2 b_{0}\right), \\
a_{1}=a_{3}=0, & b_{1}=b_{3}=0, \\
a_{2}=4, & b_{2}=-2, \\
a_{4}=-4, & b_{4}=2,
\end{aligned}
$$




$$
\begin{aligned}
u(x, t)= & \frac{1}{4}\left(-5-2 b_{0}\right) \\
& +4\left(\left(\left(1+\sec \left[x-\frac{1}{4}\left(5-6 b_{0}\right) t\right]\right.\right.\right. \\
& \left.\left.\left.+\tan \left[x-\frac{1}{4}\left(5-6 b_{0}\right) t\right]\right)^{-1 / 2}\right)^{-1}\right)^{2} \\
& -4\left(\left(1+\sec \left[x-\frac{1}{4}\left(5-6 b_{0}\right) t\right]\right)^{-1 / 2}\right)^{-1} \\
& \left.+\tan \left[x-\frac{1}{4}\left(5-6 b_{0}\right) t\right]\right)^{4}
\end{aligned}
$$

$v(x, t)=b_{0}$

$$
\begin{aligned}
& -2\left(\left(\left(1+\sec \left[x-\frac{1}{4}\left(5-6 b_{0}\right) t\right]\right.\right.\right. \\
& \left.\left.\left.+\tan \left[x-\frac{1}{4}\left(5-6 b_{0}\right) t\right]\right)^{-1 / 2}\right)^{-1}\right)^{2} \\
& +2\left(\left(\left(1+\sec \left[x-\frac{1}{4}\left(5-6 b_{0}\right) t\right]\right.\right.\right. \\
& \left.\left.\left.+\tan \left[x-\frac{1}{4}\left(5-6 b_{0}\right) t\right]\right)^{-1 / 2}\right)^{-1}\right)^{4} .
\end{aligned}
$$

(ii)

$$
\begin{gathered}
c=\frac{1}{4}\left(-1-3 b_{2}^{2}\right), \quad a_{0}=\frac{1}{4}\left(-3-b_{2}^{2}\right), \\
a_{1}=a_{3}=0, \quad b_{1}=b_{3}=b_{4}=0, \\
a_{2}=2, \quad b_{2} \neq 0, \quad a_{4}=-2, \\
u(x, t)=\frac{1}{4}\left(-3-b_{2}^{2}\right) \\
+2\left(\left(1+\sec \left[x-\frac{1}{4}\left(-1-3 b_{2}^{2}\right) t\right]\right)^{-1 / 2}\right)^{-1} \\
\left.+\tan \left[x-\frac{1}{4}\left(-1-3 b_{2}^{2}\right) t\right]\right)^{2} \\
+2\left(\left(1+\sec \left[x-\frac{1}{4}\left(-1-3 b_{2}^{2}\right) t\right]\right)^{-1}\right)^{4} \\
\left.+\tan \left[x-\frac{1}{4}\left(-1-3 b_{2}^{2}\right) t\right]\right)^{-1 / 2}
\end{gathered}
$$

$$
\begin{aligned}
v(x, t)= & -\frac{b_{2}}{2} \\
& -b_{2}\left(\left(\left(1+\sec \left[x-\frac{1}{4}\left(-1-3 b_{2}^{2}\right) t\right]\right.\right.\right. \\
& \left.\left.\left.\quad+\tan \left[x-\frac{1}{4}\left(-1-3 b_{2}^{2}\right) t\right]\right)^{-1 / 2}\right)^{-1}\right)^{2} .
\end{aligned}
$$

From the Definition 2 we have the balance term of Hirota Satsuma coupled equation by using the auxiliary equation (3) for "Case 2", is equal to -4 then we have the following system of equations

$$
\begin{aligned}
& \frac{3 a_{-4}}{2}+3 a_{-4}^{2}-6 b_{-4}^{2}=0, \\
& \left(\frac{105 a_{-3}}{128}+\frac{21}{4} a_{-4} a_{-3}-\frac{21}{2} b_{-4} b_{-3}\right)=0, \\
& \left(-\frac{a_{-1}}{16}-\frac{c a_{-1}}{2}-\frac{3}{2} a_{-1} a_{0}+3 b_{-1}\right)=0, \\
& \left(-\frac{27 a_{-4}}{4}-6 a_{-4}^{2}+\frac{9 a_{-3}^{2}}{4}+\frac{3 a_{-2}}{8}+\frac{9}{2} a_{-4} a_{-2}\right. \\
& \left.+12 b_{-4}^{2}-\frac{9 b_{-3}^{2}}{2}-9 b_{-4} b_{-2}\right)=0, \\
& \left(-\frac{225 a_{-3}}{64}-\frac{21}{2} a_{-4} a_{-3}+\frac{15}{4} a_{-3} a_{-2}\right. \\
& +\frac{15 a_{-1}}{128}+\frac{15}{4} a_{-4} a_{-1}+21 b_{-4} b_{-3} \\
& \left.-\frac{15}{2} b_{-3} b_{-2}-\frac{15}{2} b_{-4} b_{-1}\right)=0 \text {, } \\
& \left(\frac{19 a_{-4}}{2}+c a_{-4}-\frac{9 a_{-3}^{2}}{2}-\frac{3 a_{-2}}{2}-9 a_{-4} a_{-2}\right. \\
& +\frac{3 a_{-2}^{2}}{2}+3 a_{-3} a_{-1}+3 a_{-4} a_{0}-6 b_{-4} \\
& \left.+9 b_{-3}^{2}+18 b_{-4} b_{-2}-3 b_{-2}^{2}-6 b_{-3} b_{-1}\right)=0, \\
& \left(\frac{147 a_{-3}}{32}+\frac{3 c a_{-3}}{4}-\frac{15}{2} a_{-3} a_{-2}\right. \\
& -\frac{27 a_{-1}}{64}-\frac{15}{2} a_{-4} a_{-1}+\frac{9}{4} a_{-2} a_{-1} \\
& +\frac{9}{4} a_{-3} a_{0}-\frac{9 b_{-3}}{2}+15 b_{-3} b_{-2} \\
& \left.+15 b_{-4} b_{-1}-\frac{9}{2} b_{-2} b_{-1}\right)=0,
\end{aligned}
$$




$$
\begin{aligned}
& \left(-\frac{27 a_{-3}}{16}-\frac{3 c a_{-3}}{2}+\frac{13 a_{-1}}{32}+\frac{c a_{-1}}{4}\right. \\
& -\frac{9}{2} a_{-2} a_{-1}-\frac{9}{2} a_{-3} a_{0}+\frac{3}{4} a_{-1} a_{0} \\
& \left.+9 b_{-3}-\frac{3 b_{-1}}{2}+9 b_{-2} b_{-1}\right)=0 \\
& \left(-4 a_{-4}-2 c a_{-4}+\frac{7 a_{-2}}{4}+\frac{c a_{-2}}{2}\right. \\
& -3 a_{-2}^{2}-6 a_{-3} a_{-1}+\frac{3 a_{-1}^{2}}{4} \\
& -6 a_{-4} a_{0}+\frac{3}{2} a_{-2} a_{0}+12 b_{-4} \\
& \left.-3 b_{-2}+6 b_{-2}^{2}+12 b_{-3} b_{-1}-\frac{3 b_{-1}^{2}}{2}\right)=0 \\
& \left(-\frac{a_{-2}}{2}-c a_{-2}-\frac{3 a_{-1}^{2}}{2}-3 a_{-2} a_{0}+6 b_{-2}+3 b_{-1}^{2}\right)=0 \text {, } \\
& -3 b_{-4}-3 a_{-4} b_{-4}=0 \text {, } \\
& \left(-3 a_{-3} b_{-4}-\frac{105 b_{-3}}{64}-\frac{9}{4} a_{-4} b_{-3}\right)=0, \\
& \left(\frac{27 b_{-4}}{2}+6 a_{-4} b_{-4}-3 a_{-2} b_{-4}\right. \\
& \left.-\frac{9}{4} a_{-3} b_{-3}-\frac{3 b_{-2}}{4}-\frac{3}{2} a_{-4} b_{-2}\right)=0, \\
& \left(6 a_{-3} b_{-4}-3 a_{-1} b_{-4}+\frac{225 b_{-3}}{32}\right. \\
& +\frac{9}{2} a_{-4} b_{-3}-\frac{9}{4} a_{-2} b_{-3}-\frac{3}{2} a_{-3} b_{-2} \\
& \left.-\frac{15 b_{-1}}{64}-\frac{3}{4} a_{-4} b_{-1}\right)=0 \text {, } \\
& \left(-19 b_{-4}+c b_{-4}+6 a_{-2} b_{-4}-3 a_{0} b_{-4}\right. \\
& +\frac{9}{2} a_{-3} b_{-3}-\frac{9}{4} a_{-1} b_{-3}+3 b_{-2} \\
& \left.+3 a_{-4} b_{-2}-\frac{3}{2} a_{-2} b_{-2}-\frac{3}{4} a_{-3} b_{-1}\right)=0 \text {, } \\
& \left(6 a_{-1} b_{-4}-\frac{147 b_{-3}}{16}+\frac{3 c b_{-3}}{4}\right. \\
& +\frac{9}{2} a_{-2} b_{-3}-\frac{9}{4} a_{0} b_{-3} \\
& +3 a_{-3} b_{-2}-\frac{3}{2} a_{-1} b_{-2}+\frac{27 b_{-1}}{32} \\
& \left.+\frac{3}{2} a_{-4} b_{-1}-\frac{3}{4} a_{-2} b_{-1}\right)=0,
\end{aligned}
$$

$$
\begin{gathered}
\left(8 b_{-4}-2 c b_{-4}+6 a_{0} b_{-4}+\frac{9}{2} a_{-1} b_{-3}\right. \\
-\frac{7 b_{-2}}{2}+\frac{c b_{-2}}{2}+3 a_{-2} b_{-2} \\
\left.-\frac{3}{2} a_{0} b_{-2}+\frac{3}{2} a_{-3} b_{-1}-\frac{3}{4} a_{-1} b_{-1}\right)=0 \\
\left(b_{-2}-c b_{-2}+3 a_{0} b_{-2}+\frac{3}{2} a_{-1} b_{-1}\right)=0 \\
\left(\frac{27 b_{-3}}{8}-\frac{3 c b_{-3}}{2}+\frac{9}{2} a_{0} b_{-3}+3 a_{-1} b_{-2}\right. \\
\left.-\frac{13 b_{-1}}{16}+\frac{c b_{-1}}{4}+\frac{3}{2} a_{-2} b_{-1}-\frac{3}{4} a_{0} b_{-1}\right)=0 \\
\left(\frac{b_{-1}}{8}-\frac{c b_{-1}}{2}+\frac{3}{2} a_{0} b_{-1}\right)=0 .
\end{gathered}
$$

From the system of (3) we have

$$
\begin{gathered}
a_{-1}=a_{-3}=b_{-1}=b_{-3}=0, \\
b_{-2}= \pm 1, \quad b_{-4}=-\frac{b_{-2}}{2}, \quad a_{-2}=2, \\
a_{-4}=-1, \quad c=\frac{1}{4}\left(1+6 b_{-2}\right), \\
a_{0}=\frac{1}{4}\left(-1+2 b_{-2}\right),
\end{gathered}
$$

$u(x, t)$

$$
\begin{gathered}
=\frac{1}{4}+2\left(\frac{1}{\sqrt{1+i \operatorname{sech}[x-(7 / 4) t]+\tanh [x-(7 / 4) t]}}\right)^{-2} \\
-\left(\frac{1}{\sqrt{1+i \operatorname{sech}[x-(7 / 4) t]+\tanh [x-(7 / 4) t]}}\right)^{-4}
\end{gathered}
$$

$v(x, t)$

$$
\begin{aligned}
=b_{0} & +\left(\frac{1}{\sqrt{1+i \operatorname{sech}[x-(7 / 4) t]+\tanh [x-(7 / 4) t]}}\right)^{-2} \\
& -\frac{1}{2}\left(\frac{1}{\sqrt{1+i \operatorname{sech}[x-(7 / 4) t]+\tanh [x-(7 / 4) t]}}\right)^{-4},
\end{aligned}
$$


or

$$
\begin{aligned}
& u(x, t) \\
& =\frac{1}{4}+2\left(\frac{1}{\sqrt{1+\operatorname{csch}[x-(7 / 4) t]+\operatorname{coth}[x-(7 / 4) t]}}\right)^{-2} \\
& -\left(\frac{1}{\sqrt{1+\operatorname{csch}[x-(7 / 4) t]+\operatorname{coth}[x-(7 / 4) t]}}\right)^{-4}, \\
& v(x, t) \\
& =b_{0}+\left(\frac{1}{\sqrt{1+\operatorname{csch}[x-(7 / 4) t]+\operatorname{coth}[x-(7 / 4) t]}}\right)^{-2} \\
& -\frac{1}{2}\left(\frac{1}{\sqrt{1+\operatorname{csch}[x-(7 / 4) t]+\operatorname{coth}[x-(7 / 4) t]}}\right)^{-4} .
\end{aligned}
$$

Figures 1, 2, 3 and 4 gives to us 3D graphics for RLW Burgers and RLW Burgers and Hirota Satsuma coupled equations.

\section{Conclusion}

We have presented a new method and balance term definition and used it to solve the RLW Burgers and Hirota Satsuma coupled equations. In fact, this method is readily applicable to a large variety of nonlinear PDEs. First, all the nonlinear PDEs which can be solved by the other methods can be solved by our method. Second, we used only the special solutions of (3). If we use other solutions of (3), we can obtain more travelling wave solutions. Third it is a computerizable method, which allow us to perform complicated and tedious algebraic calculation on computer and so our balance term definition is effectively useful for any to chosen auxiliary equation.

\section{References}

[1] B. R. Duffy and E. J. Parkes, "Travelling solitary wave solutions to a seventh-order generalized KdV equation," Physics Letters A, vol. 214, no. 5-6, pp. 271-272, 1996.

[2] E. J. Parkes and B. R. Duffy, "Travelling solitary wave solutions to a compound KdV-Burgers equation," Physics Letters A, vol. 229, no. 4, pp. 217-220, 1997.

[3] E. J. Parkes, "Exact solutions to the two-dimensional Kortewegde Vries-Burgers equation," Journal of Physics A, vol. 27, no. 13, pp. L497-L501, 1994.

[4] L. Debnath, Nonlinear Partial Differential Equations for Scientists and Engineers, Birkhäuser, Boston, Mass, USA, 1997.

[5] A. M. Wazwaz, Partial Differential Equations: Methods and Applications, Balkema, Rotterdam, The Netherlands, 2002.

[6] W. Hereman, P. P. Banerjee, A. Korpel, G. Assanto, A. van Immerzeele, and A. Meerpoel, "Exact solitary wave solutions of nonlinear evolution and wave equations using a direct algebraic method," Journal of Physics A, vol. 19, no. 5, pp. 607-628, 1986.
[7] X.-B. Hu and W.-X. Ma, "Application of Hirota's bilinear formalism to the Toeplitz lattice-some special soliton-like solutions," Physics Letters A, vol. 293, no. 3-4, pp. 161-165, 2002.

[8] A. M. Abourabia and M. M. El Horbaty, "On solitary wave solutions for the two-dimensional nonlinear modified Kortweg-de Vries-Burger equation," Chaos, Solitons \& Fractals, vol. 29, no. 2, pp. 354-364, 2006.

[9] T. L. Bock and M. D. Kruskal, "A two-parameter Miura transformation of the Benjamin-Ono equation," Physics Letters A, vol. 74, no. 3-4, pp. 173-176, 1979.

[10] V. B. Matveev and M. A. Salle, Darboux Transformations and Solitons, Springer Series in Nonlinear Dynamics, Springer, Berlin, Germany, 1991.

[11] F. Cariello and M. Tabor, "Painlevé expansions for nonintegrable evolution equations," Physica D, vol. 39, no. 1, pp. 77-94, 1989.

[12] E. Fan and H. Zhang, "New exact solutions to a solutions to a system of coupled KdV equations," Physics Letters A, vol. 245, no. 5, pp. 389-392, 1998.

[13] E. Fan, "Auto-Bäcklund transformation and similarity reductions for general variable coefficient KdV equations," Physics Letters A, vol. 294, no. 1, pp. 26-30, 2002.

[14] E.-G. Fan, “Traveling wave solutions for nonlinear equations using symbolic computation," Computers \& Mathematics with Applications, vol. 43, no. 6-7, pp. 671-680, 2002.

[15] Y. Ugurlu and D. Kaya, "Solutions of the Cahn-Hilliard equation," Computers \& Mathematics with Applications, vol. 56, no. 12, pp. 3038-3045, 2008.

[16] I. E. Inan, "Exact solutions to the various nonlinear evolution equations," Physics Letters A, vol. 371, no. 1-2, pp. 90-95, 2007.

[17] E. Fan and H. Zhang, "A note on homogeneous balance method," Physics Letters A, vol. 246, no. 5, pp. 403-406, 1998.

[18] L. Yang, Z. Zhu, and Y. Wang, "Exact solutions of nonlinear equations," Physics Letters A, vol. 260, no. 1-2, pp. 55-59, 1999.

[19] E. Fan, "Two new applications of the homogeneous balance method," Physics Letters A, vol. 265, no. 5-6, pp. 353-357, 2000.

[20] M. L. Wang, "Solitary wave solutions for variant Boussinesq equations," Physics Letters A, vol. 199, no. 3-4, pp. 169-172, 1995.

[21] M. Wang, Y. Zhou, and Z. Li, "Application of a homogeneous balance method to exact solutions of nonlinear equations in mathematical physics," Physics Letters A, vol. 216, no. 1-5, pp. 67-75, 1996.

[22] B. Kiliç, Some methods for traveling wave solutions of the nonlinear partial differential equations and numerical analysis of these solutions [Ph.D. thesis], Firat University, 2012. 


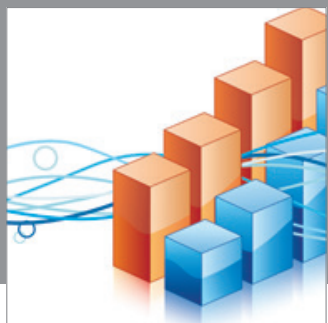

Advances in

Operations Research

mansans

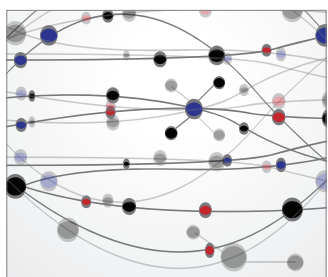

The Scientific World Journal
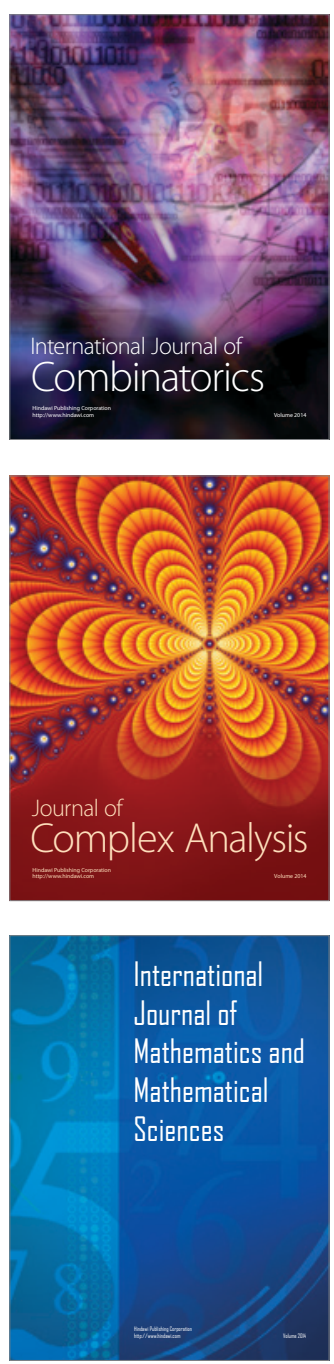
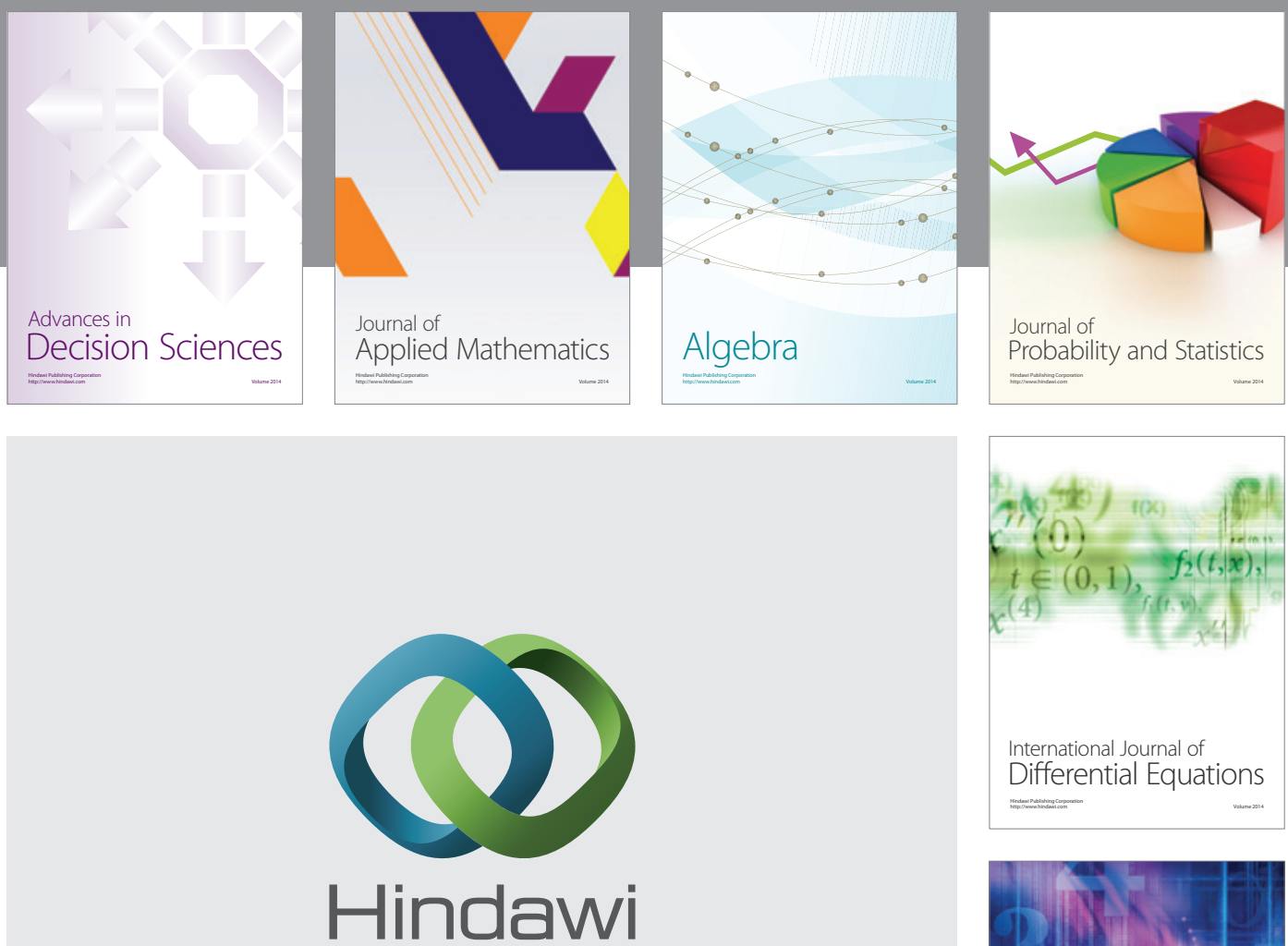

Submit your manuscripts at http://www.hindawi.com
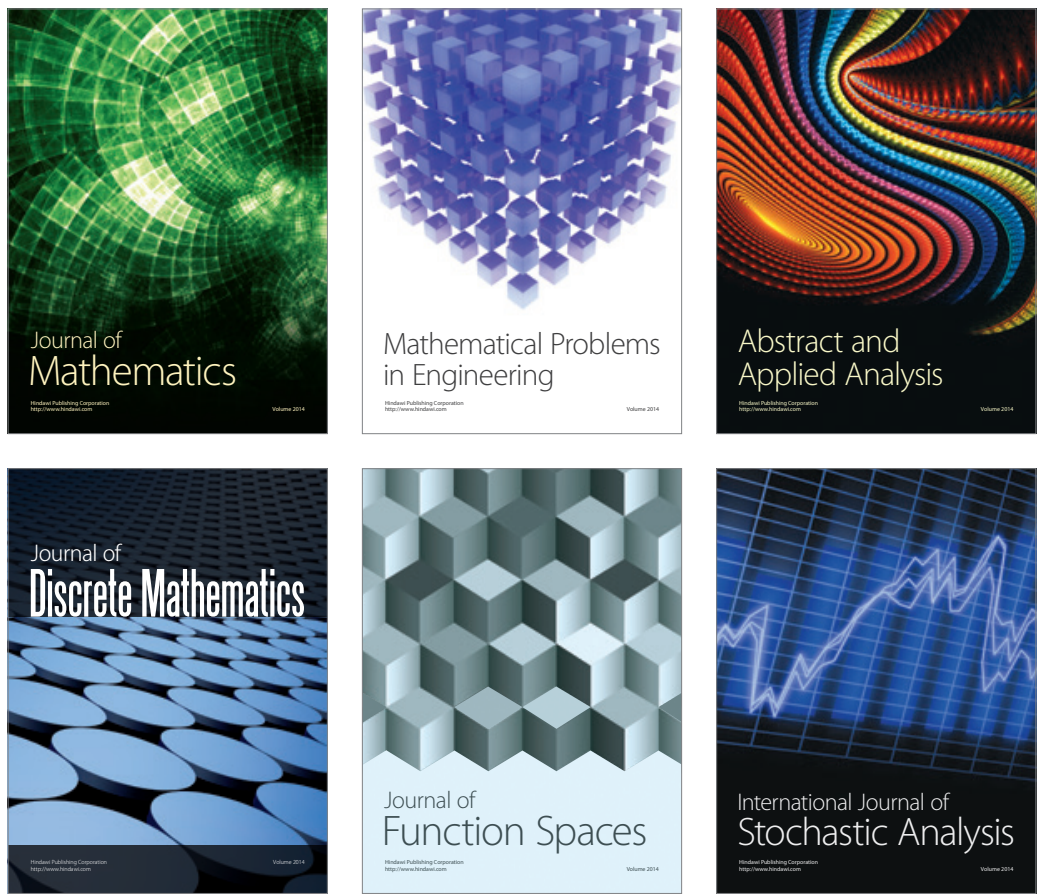

Journal of

Function Spaces

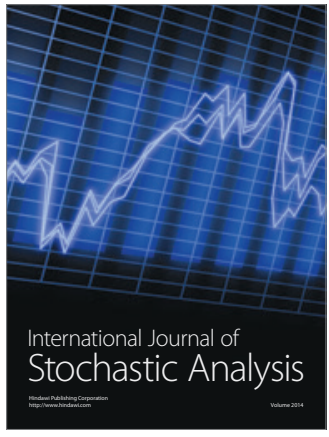

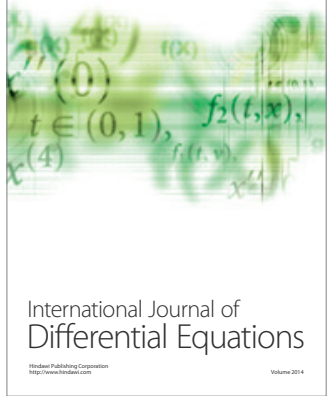
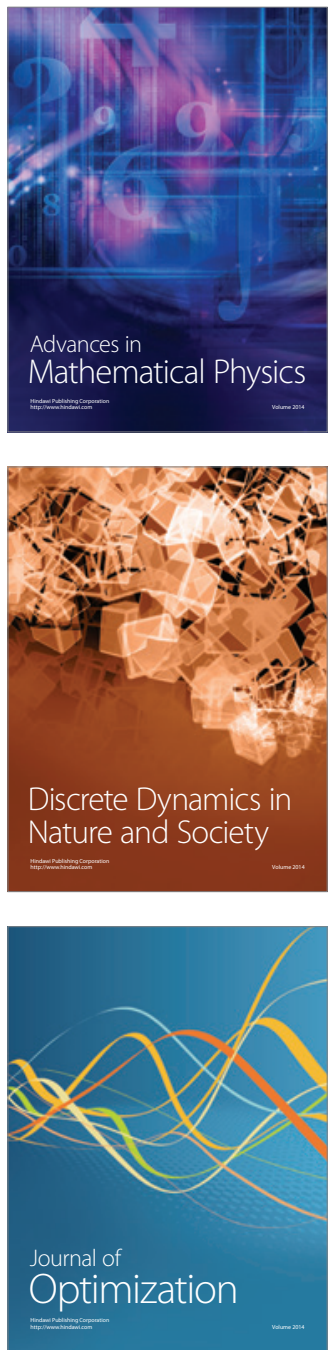